\title{
Estimulación del crecimiento en plántulas de Pinus radiata utilizando hongos ectomicorrícicos y saprobios como biofertilizantes
}

\author{
Stimulation of Pinus radiata seedling growth using ectomycorrhizal \\ and saprophytic fungi as biofertilizers
}

\author{
Daniel Chávez a*, Guillermo Pereira a , Ángela Machuca a \\ *Autor de Correspondencia: a Universidad de Concepción, Campus Los Ángeles, Departamento de Ciencias y Tecnología Vegetal, \\ Laboratorio de Biotecnología de Hongos, J. A. Coloma 0201, Los Ángeles, Chile, tel.: 56-43- 405293, danielchavez@udec.cl
}

\begin{abstract}
SUMMARY
Free-living mycorrhizal-arbuscular and saprophytic fungi have been reported to be able to promote the growth of some plants. However, the combined use of ectomycorrhizal and saprophytic fungi as growth stimulators for forest species has not been much studied to date. To study the effectiveness of different biological inoculants prepared with the ectomycorrhizal fungi Suillus luteus and Rhizopogon luteolus and the saprophytic fungi Coriolopsis rigida and Trichoderma harzianum, in both isolated and combined form, as potential biofertilizers for the growth of Pinus radiata seedlings in greenhouse conditions, $P$. radiata plants were transplanted to individual containers with pine bark composted as substrate and inoculated with sporal inoculum of ectomycorrhizal fungi and solid mycelial inoculum of saprophytic fungi produced on wheat grains. When the assays were completed (10 months), the percentage of mycorrhization and some morphological parameters of the plants were measured to determine quality indices. The biological inoculants formulated with the saprophytic fungi or with the mixture of saprophytic and ectomycorrhizal fungi stimulated plant growth compared to the controls (plants without fungal inoculation). In addition, the presence of wheat grains in the inocula promoted the stimulating effect produced by the fungi on the growth of $P$. radiata. The inoculant formulated with the $C$. rigida- $R$. luteolus mixture produced $P$. radiata plants with higher quality indices under greenhouse conditions, and its use as a potential biofertilizer represents a viable alternative in the production of forest plant species.
\end{abstract}

Key words: fungi, plant quality index, morphological growth parameters.

\section{RESUMEN}

Se ha descrito que hongos micorrícico-arbusculares y saprobios de vida libre, pueden actuar como promotores del crecimiento de algunas plantas. Sin embargo, el uso combinado de hongos ectomicorrícicos y saprobios como estimuladores del crecimiento de especies forestales ha sido poco estudiado. El objetivo de este trabajo fue estudiar la eficacia de diferentes inóculos biológicos preparados con los hongos ectomicorrícicos Suillus luteus y Rhizopogon luteolus, y los saprobios Coriolopsis rigida y Trichoderma harzianum, en forma aislada y combinada, como potenciales biofertilizantes para el crecimiento de plántulas de Pinus radiata en invernadero. Plantas de $P$. radiata se trasplantaron a recipientes individuales que contenían corteza de pino compostada como sustrato y se inocularon con esporas de los hongos ectomicorrícicos y micelio sólido de los hongos saprobios. Al finalizar los ensayos (10 meses), se midieron los porcentajes de micorrización y algunos parámetros morfológicos de las plantas para determinar índices de calidad. Los inoculantes biológicos formulados con los hongos saprobios o con la mezcla de hongos saprobios y ectomicorrícicos estimularon el crecimiento de las plantas respecto a los controles (plantas sin inoculación fúngica). Además, la presencia de granos de trigo en los inóculos favoreció el efecto estimulante producido por los hongos sobre el crecimiento de $P$. radiata. El inoculante formulado con la mezcla $C$. rigida y $R$. luteolus produjo las plantas de P. radiata con mayores índices de calidad bajo condiciones de invernadero, siendo una alternativa viable para su utilización como potencial biofertilizante en la producción de especies vegetales forestales.

Palabras clave: hongos, índices de calidad, parámetros de crecimiento, invernadero.

\section{INTRODUCCIÓN}

La asociación micorrícica es una estrategia nutricional que han desarrollado muchas plantas y algunos hongos que les asegura un beneficio mutuo. Entre dichas asociaciones destacan las ectomicorrizas, consideradas un factor fundamental para el crecimiento normal de las pináceas (Chávez et al. 2009). Los beneficios obtenidos por esta asociación son variados, destacando entre ellos la resistencia frente a agentes patógenos del suelo, la mejor adaptación al estrés hídrico, y una mayor absorción de agua y de nutrientes del suelo (Smith y Read 2008), y sobre todo, una mayor supervivencia de las plantas en campo (Duñabeitia et al. 2004). Estos beneficios varían según las condiciones ambientales en que se presenten y con la asociación particular de las especies involucradas. La utilización de plantas micorrizadas no solo ha 
facilitado la revegetación en condiciones particulares, como pueden ser la recuperación de suelos degradados o escombreras de minas y la introducción de especies exóticas en distintas partes del mundo, sino que también ha mejorado la repoblación de suelos forestales (Castellano 1996). Actualmente, es posible seleccionar el hongo que se empleará para inocular una determinada especie vegetal, el tipo de inóculo y el método de aplicación más adecuado para favorecer la formación de las ectomicorrizas y el crecimiento de las plántulas (Chávez et al. 2009). Por otra parte, estudios realizados por Donoso et al. (2008) y Arriagada et al. (2012) han determinado que hongos de vida libre, no micorrícicos, también pueden estimular el crecimiento de plantas cultivadas en vivero como es el caso de los hongos saprobios Coriolopsis rigida (Berk. et Mont.) Murrill (Basidiomycota) y Trichoderma harzianum Rifai (Ascomycota). Esta última especie es, además, reconocida por sus características como antagonista de patógeno del suelo (Donoso et al. 2008, Hohmann et al. 2011) y por ser una especie fúngica rizosférica. Sin embargo, a pesar de todos los beneficios que estos microorganismos presentan para las plantas, los viveristas aún no han incorporado el componente biológico como una herramienta de manejo en sus procesos productivos. Por otro lado, el efecto benéfico de la interacción de hongos saprobios y micorrícico-arbusculares en el crecimiento de algunas especies vegetales ha sido bien descrito en la literatura (Arriagada et al. 2009, 2012, Albrechtova et al. 2012). Sin embargo, escasos estudios han sido realizados en relación al efecto de la interacción de hongos saprobios y ectomicorrícicos en el crecimiento de especies forestales. Algunos de ellos, los trabajos de Werner et al. (2002) y Werner y Zadwórny (2003), trabajando con Pinus sylvestris, reportan la inhibición de la germinación de esporas de Trichoderma viride y T. harzianum, mostrando un enrollamiento del micelio de Laccaria laccata en conidios y esporas de Trichoderma. Zadwórmy et al. (2004), demostró que en la zonas de interacción entre el hongo ectomicorrícicos y el saprobio, las hifas de L. laccata genera una mayor pigmentación y ramificaciones cortas que crecen hacia las hifas del hongo saprobio, enrollándose alrededor de ellos. Por ello, se plantea como hipótesis que la aplicación de inóculos biológicos con hongos saprobiontes y ectomicorrícicos estimula los parámetros de crecimiento (tallo y raíz) de plantas de Pinus radiata D. Don en invernadero. De esta manera, el objetivo del presente trabajo fue estudiar el efecto que producen los inoculantes biológicos preparados con los hongos ectomicorrícicos Suillus luteus L. Gray y Rhizopogon luteolus Fr. et Nordholm y los hongos saprobios Trichoderma harzianum y Coriolopsis rigida, en forma aislada y en conjunto, sobre el crecimiento de plantas de $P$. radiata, con el fin de determinar su eficacia como biofertilizantes de especies vegetales forestales.

\section{MÉTODOS}

Especies fúngicas. Los hongos ectomicorrícicos utilizados correspondieron a dos especies pertenecientes a la colección de cultivos del Laboratorio de Biotecnología de Hongos de la Universidad de Concepción, Campus Los Ángeles: R. luteolus LBH-Rl-05 y S. luteus LBH-Sl-05, especies normalmente asociadas con plantaciones de P. radiata. Los dos hongos saprobios fueron: la especie de pudrición blanca de la madera C. rigida y la especie biocontroladora $T$. harzianum.

Producción de inóculo esporal de los hongos ectomicorrícicos. Para la producción del inóculo esporal (IE) se utilizaron 200 g de carpóforos (peso fresco) de ambas especies previamente desecados en el laboratorio. Los carpóforos se trituraron en una licuadora manual (Sindelen L2000 turbo max) conteniendo $500 \mathrm{~mL}$ de agua desionizada. Las suspensiones esporales fueron acondicionadas en recipiente de vidrio y luego se agitaron por $24 \mathrm{~h}$ a $100 \mathrm{rpm}$ en incubadora marca SI-300, con la finalidad de hidratar las esporas. El conteo esporal se realizó mediante placa de conteo Neubauer (hematocitómetro), determinando la dosis a aplicar en $1 \times 10^{7}$ esporas por planta (Rincón et al. 2001).

Producción de inóculo miceliar sólido de C. rigida y T. harzianum. Para la producción de inóculo se utilizaron como sustrato a) $150 \mathrm{~mL}$ de granos de trigo (Triticum aestivum L.) previamente hervidos durante 10 minutos con la finalidad de facilitar el crecimiento y la colonización de los hongos; y b) mezcla de vermiculita-turba en proporción 2:1, humedecida con medio de cultivo extracto de malta Merck (EM 1 \%). Los matraces Erlemeyer (250 mL) con los distintos sustratos fueron tapados y autoclavados a vapor fluente a $121{ }^{\circ} \mathrm{C}$ por 15 minutos. Bajo cámara de flujo laminar, se depositaron en la parte superior de los sustratos cinco discos de agar-micelio (5 mm de diámetro) tomados de placas con cultivo puro de las especies T. harzianum y C. rigida, según correspondiera. Los frascos en triplicado fueron incubados en oscuridad a $24 \pm 1{ }^{\circ} \mathrm{C}$ durante 10 días en condiciones estáticas. La mezcla vermiculita:turba colonizada por los hongos saprobios se utilizó como inóculo miceliar sólido (IMS) para la posterior inoculación de plantas de $P$. radiata.

Germinación e inoculación de plántulas de P. radiata con hongos ectomicorrícicos y saprobios. A las semillas de $P$. radiata seleccionadas se les aplicó un tratamiento de flotación en agua por 48 horas con la finalidad de eliminar semillas inviables. Posteriormente fueron sembradas en almacigueras con cavidades de $75 \mathrm{~mL}$ cada una, en sustrato de corteza de pino compostada. Cuando las plantas de $P$. radiata alcanzaron aproximadamente $5 \mathrm{~cm}$ de altura e iniciaron el crecimiento radicular secundario, se trasplantaron a contenedores individuales de $300 \mathrm{~mL}$ cada uno, utilizando el mismo sustrato y llevadas a la intemperie a semi-sombra. En este momento las plantas se inocularon con $10 \mathrm{~mL}$ de inóculo esporal (IE) de los hongos ectomicorrícicos inyectando con jeringa en tres puntos alrededor de la planta o con $10 \mathrm{~mL}$ de inóculo miceliar sólido (IMS) de 
los hongos saprobios, por planta, quedando estos en contacto directo con el sistema radicular de $P$. radiata. Para los tratamientos que contemplaban mezclas de inóculos fúngicos (T7-12 y T15, cuadro 1) las dosis fueron utilizadas en proporción 1:1, completando $10 \mathrm{~mL}$ de inóculo. Vale destacar que en ninguno de los tratamientos se utilizó fertilización adicional, solo riego con agua de grifo a capacidad de campo.

Evaluación de parámetros de crecimiento de plantas de P. radiata. A diez meses de la inoculación de $P$. radiata, se procedió a su evaluación, tomando en consideración crecimiento en altura (desde el cuello hasta el brote apical, cm), diámetro a la altura del cuello (DAC, mm), biomasa aérea (g) y subterránea (g) en peso seco, y porcentaje de micorrización según la metodología descrita por Brundrett et al. (1996), evaluando la cantidad de puntas micorrizadas a lo largo de las raíces sólo para los tratamientos que contemplaban hongos ectomicorrícicos. Una vez obtenidas las muestras de biomasa aérea y subterránea de las plantas de $P$. radiata, estas fueron secadas a $60{ }^{\circ} \mathrm{C}$ en estufa durante 48 horas para posteriormente ser pesadas en balanza de precisión hasta obtener peso constante, determinando así la biomasa como peso seco (g). Con los valores obtenidos se calcularon los índices de calidad de las plantas: 1) índice de Dickson (Qi) (Dickson et al. 1960), 2) índice tallo raíz (ITR) (Iverson 1984) e 3) índice de esbeltez de Schmidt-Vogt (ES) (Schmidt-Vogt 1960), según las siguientes fórmulas:

$$
\begin{aligned}
& Q i=\frac{\text { Peso_seco_total }(\mathrm{g})}{\frac{\frac{\text { Altura_tallo(cm) }}{\text { Diámetro_tallo }(\mathrm{mm})}+\mathrm{Peso}_{-} \mathrm{sec}_{-} \text {tallo }(\mathrm{g})}{\text { Peso_seco_raíces }(\mathrm{g})}} \\
& \mathrm{ITR}=\frac{\text { Peso_seco_tallo(g) }}{\text { Peso_seco_raíz }(g)} \\
& E S=\frac{\text { Diámtero_tallo(mm) }}{\frac{\text { Altura_tallo(cm) }}{10}+2}
\end{aligned}
$$

Diseño experimental y análisis estadístico. El experimento se realizó bajo un diseño completamente aleatorio, con 15 tratamientos y diez repeticiones (cuadro 1). Se incluyeron dos tratamientos testigo: planta-sustrato (corteza de pino compostada, T1) y planta-sustrato-trigo (granos de trigo hervidos y autoclavados, T2). Se midieron la altura, el diámetro, la biomasa aérea y subterránea y porcentaje de micorrización de las plantas tratadas con hongos ectomicorrícicos y control. A los valores porcentuales de micorrización se les realizaron transformaciones de variable angular dividiendo el valor porcentual por 100 y aplicando arcsen Vvalor. Los datos se sometieron a un análisis de varianza y la comparación de medias se calculó según la prueba Tukey $(P<0,05)$ (Steel y Torrie 1989). Para ello se utilizó el software Statistica versión 6.0.
Cuadro 1. Identificación de los tratamientos. Identification of treatments.

\begin{tabular}{cl}
\hline$N^{\circ}$ & \multicolumn{1}{c}{ Tratamiento (T) } \\
\hline 1 & Control (sin inóculo) \\
2 & Control Trigo (sin inóculo) \\
3 & Cr-Trigo \\
4 & Th-Trigo \\
5 & Rl \\
6 & Sl \\
7 & Cr-Trigo + Rl \\
8 & Cr-Trigo + Sl \\
9 & Th-Trigo + Rl \\
10 & Th-Trigo + Sl \\
11 & Cr-Trigo + Th-Trigo \\
12 & Rl + Sl \\
13 & Cr-vermiculita-turba \\
14 & Th-vermiculita-turba \\
15 & Cr + Th-vermiculita-turba
\end{tabular}

Cr: Coriolopsis rigida, Th: Trichoderma harzianum, Rl: Rhizopogon luteolus, Sl: Suillus luteus.

\section{RESULTADOS}

En general, los tratamientos a los que fueron sometidas las plántulas de $P$. radiata en invernadero tuvieron efectos positivos en el crecimiento en altura de las plantas (figura 1A). Con la aplicación del T7 (inoculación con la mezcla de hongos $\mathrm{Cr}$ y $\mathrm{Rl}$ ) se lograron los mayores crecimientos en altura de las plantas de $P$. radiata $(30,1 \mathrm{~cm})$, presentando diferencias significativas con los controles sin inoculación fúngica (T1 y T2) y los demás tratamientos probados. Por otro lado, los tratamientos T3 (inoculación con Cr en granos de trigo) y T8 (inoculación con mezcla Cr y Sl) también provocaron un efecto positivo en el crecimiento en altura de las plantas, significativamente diferente de los tratamientos control (T1 y T2), pero inferior al efecto de T7. Al aplicar el tratamiento control T2 (sólo granos de trigo, sin inóculo fúngico) a las plantas de $P$. radiata, se estimuló su crecimiento en altura, presentando un crecimiento significativamente mayor que en T1 (control corteza de pino compostada, sin inóculo fúngico), T5, T6 y del T12-15, que correspondieron a los tratamientos que no contenían trigo en la formulación de los inóculos (figura 1A). Respecto al diámetro a la altura del cuello (DAC) de las plantas, se observó que T7 provocó un aumento significativo en este parámetro, respecto a todos los otros tratamientos (figura 1B).

Al evaluar la cantidad de biomasa ( $g$ ) en las plantas de $P$. radiata sometidas a los diferentes tratamientos, se observó que nuevamente T7 produjo las mayores cantidades de biomasa aérea y subterránea, respecto a todos los otros 

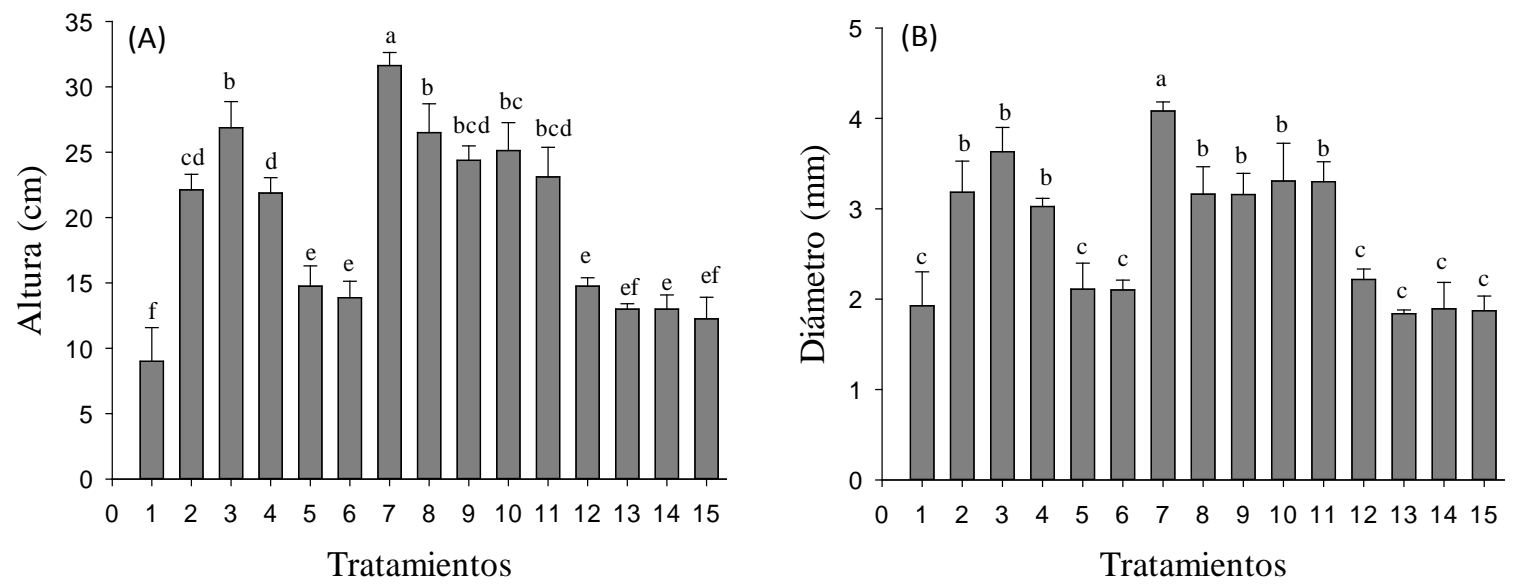

Figura 1. Atributos morfológicos de las plantas de Pinus radiata sometidas a los diferentes tratamientos T1-T15. (A) Altura (cm), (B) Diámetro a la altura del cuello $(\mathrm{mm})$. Los valores seguidos por la misma letra por tratamiento no presentan diferencias significativas $(P<0,05)$.

Morphological attributes of Pinus radiata seedlings plants subjected to treatments T1-T15 (see table 1 for treatments explanation). (A) Stem height $(\mathrm{cm})$, (B) Diameter at neck height $(\mathrm{mm})$. Values followed by the same letter per treatment present no significant differences $(P<0.05)$.

tratamientos (figura 2A). Las diferencias en la producción de biomasa aérea entre T7 y todos los demás tratamientos fueron significativas, en cambio, para la producción de biomasa subterránea $\mathrm{T} 7$ no mostró diferencias significativas con los tratamientos T2-4 y T9-11 (figura 2B). Al igual que en el crecimiento en altura y en el DAC de las plantas, el control T2 estimuló una producción de biomasa aérea y subterránea significativamente mayor que la provocada por los tratamientos T1, T5, T6 y T12-15 (figuras 2A y 2B).

Cabe destacar que en todos los tratamientos que contenían trigo (T2-4 y T7-11) de alguna forma, con o sin hongo, saprobio o ectomicorrícico, los parámetros de crecimiento de las plantas fueron significativamente mayores al control T1.

Cuando se evaluaron los diferentes índices de calidad de las plantas (cuadro 2) se pudo observar que los tratamientos donde se utilizaron granos de trigo en la formulación del inóculo presentaron los mayores valores de ITR, QI e ES (T2-4 y T7-11), destacando el tratamiento T7 con los mayores ITR $(1,33)$, QI $(0,55)$ e ES $(0,81)$ (cuadro 2). El menor ITR se obtuvo en el control T1, y el menor QI e ES con el tratamiento T13. Cabe destacar que el control T2 presentó mayores índices de calidad de las plantas que el control T1.

El grado de micorrización fue evaluado en las plantas de $P$. radiata sometidas a los diferentes tratamientos con los hongos ectomicorrícicos $R$. luteolus y S. luteus (T5-10 y T12) y en los tratamientos control (T1 y T2). Los mayores porcentajes de micorrización fueron observados en las plantas inoculadas con $R$. luteolus (T5) y con las mezclas de hongos saprobios y ectomicorrícicos (T7- 10), alcanzándose valores promedio de 17,2 \% (figura 3). Estos grados de micorrización fueron significativamente superiores a los alcanzados con S. luteus (T6) o con la combinación de los dos hongos ectomicorrícicos (T12). En todos los tratamientos se presentaron porcentajes de micorrización

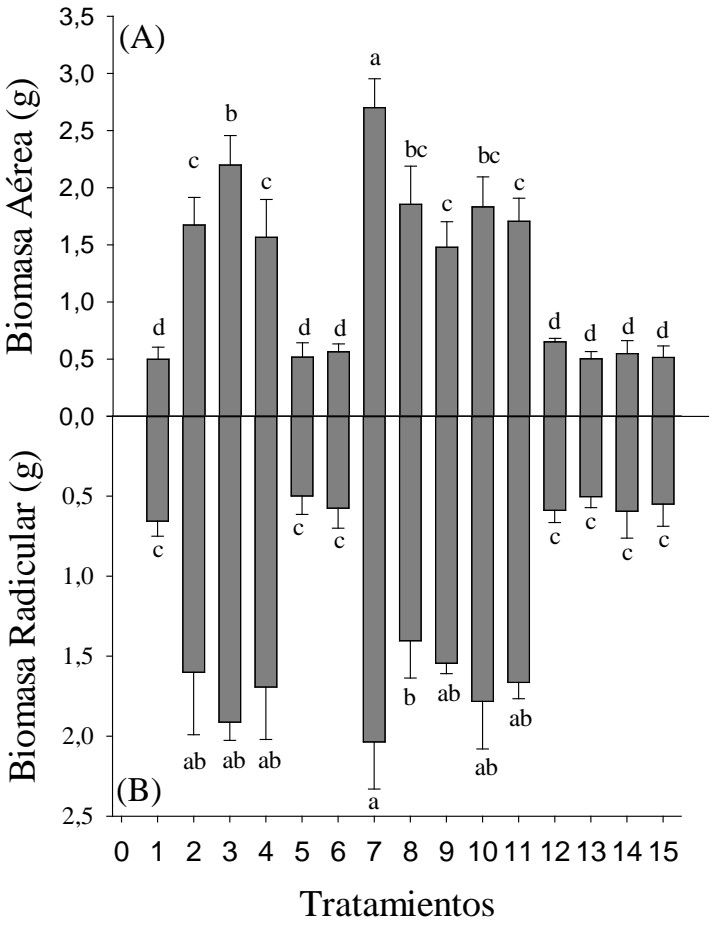

Figura 2. Producción de biomasa (peso seco) de las plantas de Pinus radiata sometidas a diferentes tratamientos. (A) Biomasa aérea (g) y (B) biomasa subterránea (g). Los valores seguidos por la misma letra por tratamiento no presentan diferencias significativas $(P<0,05)$.

Biomass production (as dry weight) by Pinus radiata seedlings subjected to different treatments. (A) Aboveground biomass (g) and (B) belowground biomass (g). Values followed by the same letter per treatment present no significant differences $(P<0.05)$. 
Cuadro 2. Índices de calidad de las plantas* de Pinus radiata sometidas a los diferentes tratamientos.

Plant quality indices for Pinus radiata plants subjected to the different treatments.

\begin{tabular}{cccc}
\hline \multirow{2}{*}{ Tratamiento } & \multicolumn{2}{c}{ Promedio \pm desviación estándar $(\mathrm{n}=10)$} \\
& ITR & QI & ES \\
\hline 1 & $0,77 \pm 0,19$ & $0,22 \pm 0,03$ & $0,66 \pm 0,10$ \\
2 & $1,06 \pm 0,23$ & $0,41 \pm 0,13$ & $0,76 \pm 0,07$ \\
3 & $1,14 \pm 0,28$ & $0,48 \pm 0,08$ & $0,78 \pm 0,08$ \\
4 & $0,92 \pm 0,07$ & $0,40 \pm 0,08$ & $0,72 \pm 0,02$ \\
5 & $1,05 \pm 0,16$ & $0,13 \pm 0,02$ & $0,61 \pm 0,07$ \\
6 & $1,02 \pm 0,29$ & $0,15 \pm 0,03$ & $0,62 \pm 0,01$ \\
7 & $1,33 \pm 0,19$ & $0,55 \pm 0,11$ & $0,81 \pm 0,06$ \\
8 & $1,33 \pm 0,22$ & $0,33 \pm 0,05$ & $0,68 \pm 0,03$ \\
9 & $0,96 \pm 0,14$ & $0,35 \pm 0,04$ & $0,71 \pm 0,06$ \\
10 & $1,04 \pm 0,13$ & $0,42 \pm 0,09$ & $0,73 \pm 0,08$ \\
11 & $1,02 \pm 0,10$ & $0,42 \pm 0,03$ & $0,77 \pm 0,07$ \\
12 & $1,12 \pm 0,18$ & $0,16 \pm 0,01$ & $0,64 \pm 0,03$ \\
13 & $1,00 \pm 0,02$ & $0,12 \pm 0,01$ & $0,56 \pm 0,01$ \\
14 & $0,96 \pm 0,24$ & $0,15 \pm 0,04$ & $0,57 \pm 0,07$ \\
15 & $0,95 \pm 0,08$ & $0,14 \pm 0,04$ & $0,58 \pm 0,05$ \\
\hline
\end{tabular}

*Qi = índice de Dickson, (ITR) = índice tallo raíz, ES = índice de esbeltez de Schmidt-Vogt.

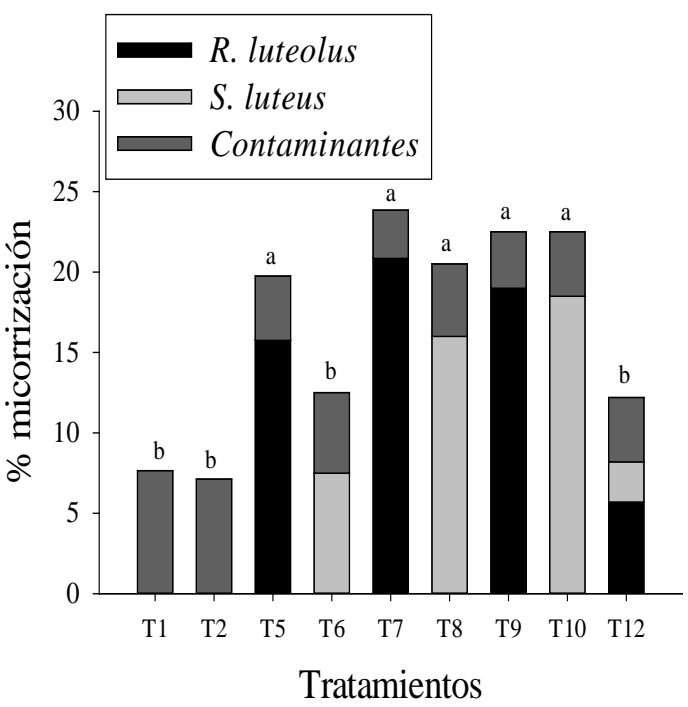

Figura 3. Porcentaje de micorrización (\%) de las plantas de $\mathrm{Pi}$ nus radiata obtenido en los distintos tratamientos. Los valores seguidos por la misma letra por especie no presentan diferencias significativas $(P<0,05)$.

Percentage of mycorrhization (\%) of Pinus radiata seedlings obtained in the different treatments. Values followed by the same letter per species present no significant differences $(P<0.05)$. entre 3 y 7,6 \% producidos por hongos contaminantes, pertenecientes a los géneros Thelephora y Hebeloma, hongos ubiquistas que se desarrollan fácilmente en invernadero. Los hongos saprobios presentaron una buena colonización y crecimiento en los sustratos y C. rigida desarrolló un micelio de color blanquecino, en cambio T. harzianum mostró un color verdoso en el sustrato, debido a la esporulación (no mostrado).

\section{DISCUSIÓN}

Los resultados del estudio indican que un manejo adecuado de los microorganismos de la rizósfera puede jugar un rol fundamental en el crecimiento y desarrollo de las plantas. Las relaciones no patogénicas entre microorganismos y una planta hospedera incrementan el crecimiento y la resistencia a diferentes tipos de estrés biótico y abiótico (Smith y Read 2008). Los mecanismos involucrados en dichos beneficios son diversos y no del todo conocidos. $\mathrm{Al}$ respecto, Pereira et al. (2007) afirman que la adecuada selección de microorganismos del suelo y su posterior manipulación, tanto en laboratorio como en vivero, pueden ser aspectos claves para lograr con éxito el establecimiento de muchas especies vegetales en campo.

Al evaluar el efecto de la inoculación en los parámetros de crecimiento se aprecia una clara variación entre los diferentes tratamientos. Se observa que en todos los tratamientos que incluyeron la presencia de granos de trigo (T2-4 y T7-11), los parámetros de crecimiento de las plantas son mejores. Cabe destacar que los granos de trigo, al ser hervidos y posteriormente esterilizados se rompen, liberando nutrientes que pueden favorecer directamente el crecimiento de las plantas o indirectamente, estimulando a los microorganismos presentes en la rizósfera como son los hongos ectomicorrícicos y saprobios. A pesar que las diferencias en los grados de micorrización no fueron estadísticamente significativas para $R$. luteolus al comparar los tratamientos T5 con T7 y T9, sí lo fueron para los tratamientos T6 con T8 y T10, que incluyeron a S. luteus. Esto sugiere que la presencia de los hongos saprobios y de los granos de trigo estimuló la micorrización de las raíces por S. luteus. De acuerdo a la clasificación dada por Tateishi et al. (2003), los porcentajes de micorrización obtenidos en este estudio se consideran bajos, siendo los mayores grados de micorrización los alcanzados con la aplicación de $R$. luteolus. Al respecto, Rincón et al. (2001), señalan que la aplicación de inóculos esporales (IE) con concentraciones de $1 \times 10^{7}$ esporas por planta, asegura inóculo suficiente para establecer buenos niveles de micorrización. Sin embargo, los resultados indican una baja incidencia en el porcentaje de micorrización alcanzado por las plantas de $P$. radiata cuando se utilizó IE, independiente de la especie ectomicorrícica probada. Ensayos similares en Pseudotsuga menziesii (Mirb.) Franco y P. radiata demostraron la influencia positiva de Rhizopogon sp. en el desarrollo aéreo de las plantas utilizando IE (Chu-Chou y Grace 1985), 
resultados que no concuerdan con los presentados en este trabajo, posiblemente, debido a problemas de inviabilidad esporal al momento de la inoculación. Otras experiencias relacionadas con micorrización de Pinus ponderosa Dougl. ex Laws en vivero a raíz cubierta, con esporas de $R$. roseolus (Barroetaveña et al. 2012) lograron altos porcentajes de micorrización (34,9\%), pero los valores de los parámetros morfométricos de las plantas no superaron a los testigos. Baltasar et al. (2007) trabajaron con las mismas especies vegetal y fúngica concluyeron que, ante una disminución acentuada en las dosis de fertilizante al final de la etapa de rustificación en vivero (periodo de letargo o endurecimiento de plantas), aumenta significativamente la micorrización de $P$. ponderosa, pero que los niveles de fertilización de nitrógeno, fósforo y potasio (alto, medio y bajo) utilizados, así como los distintos tiempos de inoculación (dos semanas y cuatro meses de la siembra) no produjeron diferencias significativas en los porcentajes de micorrización, bajo el esquema de producción tradicional. En cuanto a la presencia de hongos no deseados (contaminantes) en los sistemas radicales de las plantas de $P$. radiata, esto era esperable ya que se trabajó en condiciones de invernadero abierto. Sin embargo, cabe resaltar que a pesar de ser hongos no deseados para esta investigación, constituyen organismos benéficos para el crecimiento de las plantas (Pera y Parladé 2005).

Por otra parte, queda en evidencia que cuando se trata del crecimiento en altura y producción de biomasa aérea por las plantas, el resultado de la adición de C. rigida al sistema (T3) fue mejor que usar solamente granos de trigo (control T2). La adición conjunta de C. rigida y R. luteolus (T7) al sistema fue todavía mejor que T3, no sucediendo lo mismo con el otro hongo saprobio, T. harzianum, a pesar de su conocido efecto benéfico como promotor del crecimiento de especies vegetales descrito en la literatura (Donoso et al. 2008, Hohmann et al. 2011). La especie C. rigida produce efectos significativos en el crecimiento en altura y biomasa aérea de $P$. radiata de forma individual o en las distintas mezclas con los hongos ectomicorrícicos, respecto al control $\mathrm{T} 2$.

Cuando se evaluaron los diferentes índices de calidad de las plantas, a los diez meses, se pudo corroborar que los mayores valores de los diferentes índices se obtuvieron con el tratamiento T7. No obstante, los valores de ITR obtenidos en todos los tratamientos $(0,77$ a 1,33$)$ fueron inferiores a los requeridos para definir una planta de buena calidad que, según Iverson (1984), deben encontrarse entre 1,5 y 2 , dependiendo de la especie analizada. Al analizar los valores de QI se pudo apreciar que todos los tratamientos que contenían granos de trigo en la formulación del inóculo presentaron índices adecuados $(0,33-0,55)$ para definir una planta de buena calidad. Hunt (1990) recomienda un índice de Dickson superior a 0,2 para plantas de Pinus spp. y señala que un valor inferior a 0,15 en Abies spp. y Pinus spp. puede significar problemas en el establecimiento de las plantas en campo, debido a un desequilibrio entre la parte aérea evapotranspirante y la radical absorbente. Por otro lado, Dickson et al. (1960) consideran que cuanto más alto es el valor QI mejor es la calidad de la planta. En este estudio los mayores valores de QI $(0,55)$ se alcanzaron cuando las plantas fueron sometidas a tratamiento con una mezcla de los hongos $C$. rigida y R. luteolus (T7). En cuanto al índice de esbeltez (ES), un valor de 1 se considera adecuado para plantas normales de $P$. ponderosa (Dengler et al. 1990). Sin embargo, en el presente estudio los valores fueron levemente inferiores para algunos tratamientos, alcanzándose el mayor índice de ES con el tratamiento T7 $(0,88)$. Cabe destacar que en la mayoría de los trabajos donde se evalúa la calidad de las plantas en vivero se trabaja bajo programas de fertirriego, donde las plantas están por periodos prolongados (8-12 meses) expuestas a condiciones ideales. Para el presente estudio en cambio, se trabajó con la aplicación de inóculos biológicos sin ningún apoyo de fertilizantes, solo riego con agua a capacidad de campo. Por ello, los resultados de crecimiento de las plántulas de $P$. radiata obtenidos con el tratamiento T7 resultan promisorios y deberían conducir a disminuir la aplicación de fertilizantes comerciales en viveros forestales, para dar paso a la utilización de inóculos biológicos como biofertilizantes. Sin embargo, posteriores investigaciones son necesarias para corroborar el efecto benéfico de la aplicación de mezclas de hongos saprobios y ectomicorrícicos en el crecimiento de especies de interés forestal, además de evaluar el efecto combinado de inóculos biológicos con aplicación moderada de fertilizantes.

\section{CONCLUSIONES}

La interacción del hongo saprobio C. rigida y del hongo ectomicorrícico $R$. luteolus es la más beneficiosa para la estimulación del crecimiento de plantas de P. radiata bajo condiciones de invernadero.

El manejo de microorganismos rizosféricos y saprobios para la formulación de inóculos biológicos y su potencial uso como biofertilizantes demuestran ser alternativas viables de implementar en la producción de plantas forestales como P. radiata.

\section{AGRADECIMIENTOS}

Los autores agradecen al Dr. César Arriagada de la Universidad de la Frontera, Temuco, y al Departamento de Ciencias y Tecnología Vegetal de la Universidad de Concepción. Daniel Chávez Agradece el apoyo de la Comisión Nacional de Investigación Científica y Tecnológica CONICYT por la Becas de Postgrado asignadas N²1110038.

\section{REFERENCIAS}

Albrechtova J, A Latr, L Nedorost, R Pokluda, K Posta, M Vosatka. 2012. Dual inoculation with mycorrhizal and saprotrophic fungi applicable in sustainable cultivation improves 
the yield and nutritive value of onion. The Science World Journal 2012: 1-8.

Arriagada C, I Sampedro, I García-Romera, J Ocampo. 2009. Improvement of growth of Eucalyptus globulus and soil biological parameters by amendment with sewage sludge and inoculation with arbuscular mycorrhizal and saprobe fungi. Science of the total environment 407(17): 4799-4806.

Arriagada C, D Manquel, P Cornejo, J Soto, I Sampedro, J Ocampo. 2012. Effects of the co-inoculation with saprobe and mycorrhizal fungi on Vaccinium corymbosum growth and some soil enzymatic activities. Journal Soil Science Plant Nutrition 12(2): 283-294.

Baltasar M, C Barroetaveña, M Rajchenberg. 2007. Influencia del régimen de fertilización y del momento de inoculación en la micorrización de Pinus ponderosa en la etapa de vivero. Bosque 28(3): 226-233.

Barroetaveña C, VN Bassani, M Rajchenberg. 2012. Inoculación micorrícica de Pinus ponderosa en la Patagonia Argentina: colonización de las raíces, descripción de morfotipos y crecimiento de las plántulas en vivero. Bosque 33 (2): 163-169.

Belanger R, N Dufuor, J Caron, N Benhamou. 1995. Chronological events associated with the antagonistic properties of Trichoderma harzianum against Botrytis cinerea: Indirect evidence for sequential role of antibiotics and parasitism. Biocontrol Science Technology 5(1): 41-54.

Brundrett M, N Bougher, T Grove, N Malajczuk. 1996. Working with mycorrhizas in forestry and agriculture. Monograph 32. Canberra, Australia. Australian Center for International Agricultural Research. 374 p.

Castellano M. 1996. Outplanting performance of mycorrhizal inoculated seedlings. In Mukerji KG. ed. Concepts in Mycorrhizal Research. Kluwer The Netherlands. Academic Publ. p. 223-301.

Chávez D, G Pereira, A Machuca. 2009. Efecto de tipos de inóculos de tres especies fúngicas en la micorrización controlada de plántulas de Pinus radiata. Bosque 30(1): 4-9.

Chu-Chou M, J Grace. 1985. Comparative efficiency of the mycorrhizal fungi Laccaria laccata, Hebeloma crustuliniforme and Rhizopogon species on growth of radiata pine seedlings. New Zealand Journal of Botany 23(3): 417-424.

Dengler A, E Röhrig, HA Gussone. 1990. Waldbau auf ökologischerGrundlage. Zweiter Band. $6^{\mathrm{a}}$ ed. Hamburg y Berlin, Alemania. Paul Parey. 314 p.

Dickson A, A Leaf, I Hosner. 1960. Quality appraisal of White Spruce and White Pine seedlings stock in nurseries. Forestry Chronicle 36(1): 10-13.

Donoso E, G Lobos, N Rojas. 2008. Efecto de Trichoderma harzianum y compost sobre el crecimiento de plántulas de $\mathrm{Pi}$ nus radiata en vivero. Bosque 29 (1): 52-57.

Duñabeitia MK, S Hormilla, J Garcia-Plazaola, K Txarterina, U Arteche, JM Becerril. 2004. Differential responses of three fungal species to environmental factors and their role in the mycorrhization of Pinus radiata D. Don. Mycorrhiza 14: 11-18.

Hohmann P, E Jones, A Hill, A Stewart. 2011. Understanding Trichoderma in the root system of Pinus radiata: associations between rhizosphere colonization and growth promotion for commercially grown seedlings. Fungal biology 115(8): 759-767.

Hunt GA. 1990. Effect of styroblock design and copper on morphology of conifer seedlings. In Rose R, SJ Campbell, TD Landis eds. Proceedings, Western Forest Nursery Association. Fort Collins, CO USA. p. 218-222.

Iverson R. 1984. Planting stock selection: Meeting biological needs and operational realities. In Duryea ML, TD Landis eds. Forest nursery manual. Oregon, State University. p. 261-266.

Onguene NA, TW Kuyper. 2002. Importance of the ectomycorrhizal network for seedling survival and ectomycorrhiza formation in rain forests of south Cameroon. Mycorrhiza 12 (1): 13-17.

Pera J, J Parladé. 2005. Inoculación controlada con hongos ectomicorrícicos en la producción de planta destinada a repoblaciones forestales: estado actual en España. Investigación Agraria: Sistemas de Recursos Forestales 14(3): 419-433.

Pereira G, J Herrera, A Machuca, M Sánchez. 2007. Efecto del $\mathrm{pH}$ sobre el crecimiento in vitro de hongos ectomicorrícicos recolectados de plantaciones de Pinus radiata. Bosque 28(3): 215-219.

Rincón AM, IF Alvarez, J Pera. 2001. Inoculation of containerized Pinus pinea L. seedlings with seven ectomycorrhizal fungi. Mycorrhiza 11(6): 265-271.

Schmidt-Vogt H. 1980. Characterization of plant material, IUFRO Meeting. S1.05-04. In Röhring E, Gussone HA. Waldbau. Zweiter band. Sechste Auflage, Neubearbeitet. Hamburg and Berlin, 1990. 314 p.

Sid Ahmed A, M Ezziyyani, C Pérez, ME Candela. 2003. Effect of chitin on biological control activity of Bacillus spp. and Trichoderma harzianum against root rot disease in pepper (Capsicum annuum) plants. European Journal of Plant Pathology 109 (6):633-637.

Smith SE, DJ Read. 2008. Mycorrhizal symbiosis $3^{\text {th }}$ ed. Cambridge, Inglaterra. Academic Press. 605 p.

Steel R, J Torrie. 1989. Bioestadística: Principios y procedimientos. México DF, México. 2a ed. McGraw Hill. 662 p.

Tateishi T, K Yokoyama, N Konho, H Marumotot. 2003. Estimation of mycorrhizal colonization of the roots of Oak seedlings inoculated with an ectomycorrhizal fungus Laccaria amethystea. Journal of Soil Science and Plant Nutrition 49 (4): 641-645.

Werner A, Zadworny M, Idzikowska K. 2002. Interaction between Laccaria laccata and Trichoderma virens in co-culture and in the rhizosphere of Pinus sylvestris grown in vitro. Mycorrhiza 12:139-145. 
